\title{
Chaos synchronization of a fractional nonautonomous system
}

\begin{abstract}
In this paper we investigate the dynamic behavior of a nonautonomous fractional-order biological system. With the stability criterion of active nonlinear fractional systems, the synchronization of the studied chaotic system is obtained. On the other hand, using a Phase-Locked-Loop (PLL) analogy we synchronize the same system. The numerical results demonstrate the effectiveness of the proposed methods.
\end{abstract}

Keywords: Chaos; Fractional-order system; Active control; PLL; Synchronization

Zakia Hammouch, Toufik Mekkaoui: E3MI Group Department of Mathematics,, FSTE Moulay Ismail University,, BP 509 Boutalamine Errachidia 52000, Morocco, E-mail: hammouch.zakia@gmail.com, toufik-mekkaoui@tyahoo.fr

\section{Introduction}

The study of nonlinear dynamical systems and chaos have become a subject of great interest and it has attracted enormous research interest after the earlier numerical demonstration of chaos by Lorenz [16]. Chaotic systems are associated with complex dynamical behaviors that possess some special features including bounded trajectories with positive Lyapunov exponents; and sensitive dependence on initial conditions. The study of chaotic dynamics has found applications in various fields of scientific and engineering disciplines, including meteorology, physics, chemistry, engineering, economics, biology, etc [24? ]. On the other hand, the development of models based on fractional-order differential systems has recently received growing attention in the investigation of dynamical systems. The interest in the study of fractional-order nonlinear systems lies in the fact that fractional derivatives provide an excellent tool for the description of memory and hereditary properties, which are not taken into account in the classical integer-order models. Further fractional versions of many systems were investigated $([3,11,12,24,30])$. Owing to its interesting applications in secure communication of analog and digital signals, cryptographic systems and control proccessing, time series analysis, modelling brain and cardiac rhythmic activity as well as earthquake dynamics [25], chaos synchronization has been an interesting research area since the pioneering work of Carroll and Peccora [23]. Nowadays, different techniques and methods have been proposed to achieve chaos synchronization such as linear and nonlinear feedback control [17], adaptive control [29], sliding mode control [10], backstepping nonlinear control approach [21], etc. In this work we consider a nonautonomous fractional-order dynamical system arising in biology $[5,14,15]$. The dynamical behaviour of the system is studied. Using two synchronization schemes we force the slave trajectories to track the master trajectories.

The remainder of the paper is organized as follows: After this introduction, preliminaries regarding fractional calculus and numerical scheme are described in Section 2. In Section 3, the fractional-ordered model is described and its dynamical behaviour is studied. In Section 4 synchronization via two methods is presented, numerical simulations of the results are presented and discussed. Finally, the paper is concluded in Section 5. 


\section{Preliminaries}

\subsection{Fractional Calculus}

We briefly recall here some basic necessary tools on fractional calculus. For more details on the subject and applications, we refer the reader to the references [4] [19] and [26].

Definition 1. A real function $f(x), x>0$, is said to be in the space $C_{\mu}, \mu \in \mathbb{R}$ if there exits a real number $\lambda>\mu$ such that $f(x)=x^{\lambda} g(x)$, where $g(x) \in C[0, \infty)$ and it is said to be in the space $C_{\mu}^{m}$ if and only if $f^{(m)} \in C_{\mu}$ for $m \in N$.

Definition 2. The Riemann-Liouville fractional integral operator of order $q$ of a real function $f(x) \in C_{\mu}, \mu \geq$ -1 , is defined as

$$
J^{q} f(x)=\frac{1}{\Gamma(q)} \int_{0}^{x}(x-t)^{q-1} f(t) d t, \quad q>0, x>0 \quad \text { and } \quad J^{0} f(x)=f(x) .
$$

The the operators $J^{q}$ has some proprieties, for $q, p, \xi \geq 0$ :

$-J^{q} J^{p} f(x)=J^{q+p} f(x)$,

- $J^{q} J^{p} f(x)=J^{p} J^{q} f(x)$

- $J^{q} \chi^{\xi}=\frac{\Gamma(\xi+1)}{\Gamma(q+\xi+1)} \chi^{q+\xi}$.

Where $\Gamma$ is the Euler's Gamma function.

Definition 3. The Caputo fractional derivatives $D^{q}$ of a function $f(x)$ of any real number $q$ such that $m-1<$ $q \leq m, m \in N$, for $x>0$ and $f \in C_{-1}^{m}$ in the terms of $J^{q}$ is defined as

$$
D^{q} f(x)=J^{m-q} D^{m} f(x)=\frac{1}{\Gamma(m-q)} \int_{0}^{x}(x-t)^{m-q-1} f^{(m)}(t) d t,
$$

and has the following proprieties for $m-1<q \leq m, m \in N, \mu \geq-1$ and $f \in C_{\mu}^{m}$,

- $\quad D^{q} J^{q} f(x)=f(x)$,

- $J^{q} D^{q}=f(x)-\sum_{k=0}^{m-1} f^{(k)}\left(0^{+}\right) \frac{x^{k}}{k !}$, for $x>0$.

Definition 4. The Laplace transform of the Caputo fractional derivative is given by

$$
L\left\{D^{q} f(t)\right\}=s^{q} L\{f(t)\}-\sum_{k=0}^{m-1} s^{q-1-k} f^{(k)}(0),
$$

where $L$ means the Laplace transform and $s$ is a complex variable. Upon considering the initial conditions to zero, Eq.(3) reduces to

$$
L\left\{D^{q} f(t)\right\}=s^{q} L\{f(t)\} .
$$

\section{Stability conditions}

Theorem 1 (see [18]). For a given fractional order system

$$
D^{q} X(t)=A X(t), \quad X(0)=X_{0},
$$


with $X(t)=\left(x_{1}, x_{2}, \ldots, x_{n}\right)^{T} \in \mathbb{R}^{n}, A=\left(a_{i j}\right) \in \mathbb{R}^{n x m}$ and $q=\left(q_{1}, q_{2}, \ldots, q_{n}\right)^{T}$, where $0<q_{i} \leq 2(i=$ $1,2, \ldots, n)$. Assume $m$ to be the lowest common multiple of the denominators $u_{i}$ of $q_{i}$ satisfying

$$
q_{i}=\frac{v_{i}}{u_{i}},\left(u_{i}, v_{i}\right)=1, u_{i}, v_{i} \in \mathbb{Z}_{+} .
$$

Set $\gamma=\frac{1}{m}$. Define the following characteristic equation

$$
\operatorname{det}\left(\operatorname{diag}\left(\lambda^{m q_{1}}, \lambda^{m q_{2}}, \ldots, \lambda^{m q_{n}}\right)-A\right)=0 .
$$

Then the steady state of system (5) is globally asymptotically stable if all roots $\lambda_{i}$ of equation (6) satisfy

$$
\left|\arg \left(\lambda_{i}\right)\right|>\frac{\pi}{2} \gamma
$$

For studying the stability of fractional order systems, we will need the following final value theorem.

Theorem 2. Let $F(s)$ be the Laplace transform of a function $f(t)$. If all poles of $s F(s)$ are in the open left half plane, then

$$
\lim _{t \rightarrow \infty} f(t)=\lim _{s \rightarrow 0} s F(s) .
$$

\subsection{Adams-Bashforth (PECE) algorithm}

Consider the fractional-order initial value problem

$$
\left\{\begin{array}{l}
D_{t}^{q} y=f(t, y(t)) \quad 0 \leq t \leq T, \\
y^{(k)}(0)=y_{0}^{(k)}, \quad k=0,1, \ldots, m-1 .
\end{array}\right.
$$

It is equivalent to the Volterra integral equation

$$
y(t)=\sum_{k=0}^{[q]-1} y_{0}^{(k)} \frac{t^{k}}{k !}+\frac{1}{\Gamma(q)} \int_{0}^{t}(t-s)^{q-1} f(s, y(s)) d s .
$$

Diethelm et al. have given a predictor-correctors scheme (see [6][7]), based on the Adams-BashforthMoulton algorithm to integrate Equation (9). By applying this scheme to the fractional-order system (8), and setting

$$
h=\frac{T}{N}, \quad t_{n}=n h, \quad n=0,1, \ldots, N,
$$

Equation (9) can be discretized as follows:

$$
y_{h}\left(t_{n+1}\right)=\sum_{k=0}^{[q]-1} y_{0}^{(k)} \frac{t^{k}}{k !}+\frac{h^{q}}{\Gamma(q+2)} f\left(t_{n+1}, y_{h}^{p}\left(t_{n+1}\right)\right)+\frac{h^{q}}{\Gamma(q+2)} \sum_{j=0}^{n} a_{j, n+1} f\left(t_{j}, y_{h}\left(t_{j}\right)\right),
$$

where

$$
\left\{\begin{array}{l}
n^{q+1}-(n-q)(n+1)^{q}, j=0, \\
(n-j+2)^{q+1}+(n-j)^{q+1}-2(n-j+1)^{q+1}, \quad 1 \leq j \leq n \\
1, \quad j=n+1,
\end{array}\right.
$$

and the predictor is given by

$$
y_{h}^{p}\left(t_{n+1}\right)=\sum_{k=0}^{[q]-1} y_{0}^{(k)} \frac{t^{k}}{k !}+\frac{1}{\Gamma(q)} \sum_{j=0}^{n} b_{j, n+1} f\left(t_{j}, y_{h}\left(t_{j}\right)\right),
$$


where $b_{j, n+1}=\frac{h^{q}}{q}((n+1)-j)^{q}-(n-j)^{q}$. The error estimate of the above scheme is

$$
\max _{j=0,1, . ., N}\left\{\left|y\left(t_{j}\right)-y_{h}\left(t_{j}\right)\right|\right\}=O\left(h^{p}\right),
$$

in which $p=\min (2,1+q)$.

\subsection{Synchronization of two fractional order non-autonomous systems}

Consider the master-slave synchronization scheme of two non-autonomous fractional order systems

$$
\left\{\begin{array}{l}
D_{t}^{q} X_{1}=f\left(t, X_{1}(t)\right) \quad \text { (Master) } \\
D_{t}^{q} X_{2}=g\left(t, X_{2}(t)\right)+U(t) \quad \text { (Slave). }
\end{array}\right.
$$

Where $q$ is the fractional-order of derivation, $X_{1}, X_{2} \in \mathbb{R}^{n}$ are the states of the master and the slave systems respectively and $f, g: \mathbb{R}^{n} \rightarrow \mathbb{R}^{n}$ are the vector fields of the master and the slave systems respectively.

The main goal is to take a suitable line control function $U(t)=\left(u_{1}(t), \ldots, u_{n}(t)\right)$ such that the states of the master and the slave systems are synchronized, i.e, $\lim _{t \rightarrow \infty}\left\|X_{1}-X_{2}\right\|=0$, where $\|\cdot\|$ denotes the Euclidean norm.

\section{A nonautonomous fractional-order biological system}

We give a brief description of the biological model that we will study in the present paper; it consists of enzyme-substrate system with ferroelectric behaviour in brain waves $[8,9,14,15]$. It is described by the second order nonautonomous differential equation of the activated enzyme molecules $[13,21]$

$$
\ddot{x}-\mu\left(1-x^{2}+\alpha x^{4}-\beta x^{6}\right) \dot{x}+\chi=E \cos (\omega t),
$$

where an overdot represents differentiation with respect to time $t, \alpha$ is the parameter of nonlinearity and $x$ is the concentration of the biological system. The quantities $\alpha, \beta$ are positive real parameters while $E$ and $\omega$ are respectively the amplitude and the frequency of the external excitation.

In [21], the authors convert Equation (14) to the following system

$$
\left\{\begin{array}{l}
\dot{x}=y, \\
\dot{y}=\mu\left(1-x^{2}+\alpha x^{4}-\beta x^{6}\right) y-x+E \cos (\omega t) .
\end{array}\right.
$$

They proposed recursive and adaptive backstepping nonlinear controllers to control and synchronize the biological system (15).

In this paper, we consider the fractional commensurate ordered model of (15), which takes the form

$$
\left\{\begin{array}{l}
D_{t}^{q} x=y, \\
D_{t}^{q} y=\mu\left(1-x^{2}+\alpha x^{4}-\beta x^{6}\right) y-x+E \cos (\omega t),
\end{array}\right.
$$

where $q \in(0,1], \alpha=2.55, \beta=1.70, \mu=2.001, \omega=3.465$ and $E=8.27$.

It is easy to check that (16) possesses one equilibrium point $(0,0)$. The Jacobian matrix at this point is

$$
\left.J\right|_{(0,0)}=\left(\begin{array}{cc}
0 & 1 \\
-1 & \mu
\end{array}\right)
$$


and the corresponding eigenvalues are

$$
\lambda_{1}=\frac{\mu+\sqrt{\mu^{2}-4}}{2}, \quad \lambda_{2}=\frac{\mu-\sqrt{\mu^{2}-4}}{2} .
$$

Since $\lambda_{1}$ is positive, then

$$
\arg \lambda_{1}<\frac{\pi}{2} q
$$

Therefore the equilibrium point $O$ is unstable.

Fig. 1 depicts the chaotic behaviour of system (16) by considering : $q=0.9$ and the initial conditions : $(x(0), y(0))=(0,1)$.

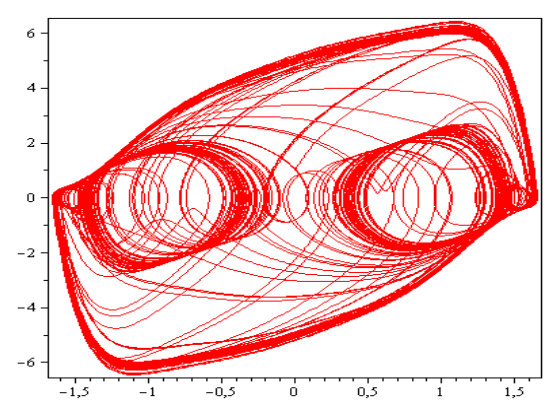

Fig. 1. Chaotic phase portrait of (16) for $q=0.9, \alpha=2.55, \beta=1.70, \mu=2.001, \omega=3.465, E=8.27$ and $(x(0), y(0))=(0,1)$.

\section{Synchronisation of the NAFOBS}

In this section, we aim to achieve the chaos synchronization of two identical fractional-order biological systems by using two methods, namely the active control synchronization and the phase-locked-loop analogy. A master-slave synchronization scheme is illustrated in Fig.2.

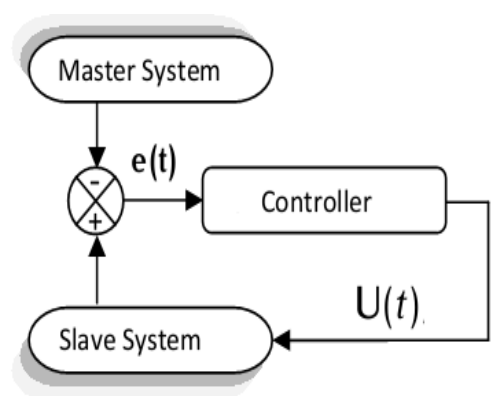

Fig. 2. The master-slave synchronization scheme.

\subsection{Active control method}

Chaos synchronization using active control was proposed by Bai and Lonngren [1] and has recently been widely accepted as an efficient technique for the synchronization of chaotic systems.

To demonstrate the effectiveness of the used methods, we will give some numerical simulations which are conducted by a PECE scheme, using Maple 17 software with a time-step $h=10^{-2}$. For convenience, the parameters of system (16) are specified as $\alpha=2.55, \beta=1.70, \mu=2.001, \omega=3.465, E=8.27$. 
Now consider the master-slave synchronization scheme of two non-autonomous identical fractional-order systems as follows

$$
\text { (Master) }\left\{\begin{array}{l}
D_{t}^{q} x_{1}=y_{1}, \\
D_{t}^{q} y_{1}=\mu\left(1-x_{1}^{2}+\alpha x_{1}^{4}-\beta x_{1}^{6}\right) y_{1}-x_{1}+E \cos (\omega t), \\
\left(x_{1,0}, y_{1,0}\right) .
\end{array}\right.
$$

and

$$
\text { (Slave) }\left\{\begin{array}{l}
D_{t}^{q} x_{2}=y_{2}+u_{1}(t), \\
D_{t}^{q} y_{2}=\mu\left(1-x_{2}^{2}+\alpha x_{2}^{4}-\beta x_{2}^{6}\right) y_{2}-x_{2}+E \cos (\omega t)+u_{2}(t), \\
\left(x_{2,0}, y_{2,0}\right) .
\end{array}\right.
$$

Where $u_{1}$ and $u_{2}$ are unknown active control functions to be determined. Note that the initial conditions $\left(x_{1,0}, y_{1,0}\right)$ and $\left(x_{2,0}, y_{2,0}\right)$ are different and we want to synchronize the signals in spite of discrepancy between the initial conditions. So let us define the error vector $\mathbf{e}(\mathbf{t})$ as

$$
\left\{\begin{array}{l}
e_{1}=x_{2}-x_{1} \\
e_{2}=y_{2}-y_{1}
\end{array}\right.
$$

The substraction of (17) from (18) and the use of (19) gives

$$
\left\{\begin{array}{l}
D^{q} e_{1}=e_{1}+u_{1}, \\
D^{q} e_{2}=-e_{1}-\mu\left(y_{2} x_{2}^{2}-y_{1} x_{1}^{2}\right)+\mu \alpha\left(y_{2} x_{2}^{4}-y_{1} x_{1}^{4}\right)-\mu \beta\left(y_{2} x_{2}^{6}-y_{1} x_{1}^{6}\right)+u_{2}(t) .
\end{array}\right.
$$

Let

$$
\left\{\begin{array}{l}
u_{1}=-2 e_{1}-e_{2}, \\
u_{2}=e_{1}-2 \mu e_{2}+\mu\left(y_{2} x^{2}-y_{1} x_{1}^{2}\right)-\mu\left(y_{2} x_{2}^{4}-y_{1} x_{1}^{4}\right)+\mu \beta\left(y_{2} x_{2}^{6}-y_{1} x_{1}^{6}\right) .
\end{array}\right.
$$

Thus, the fractional-order error dynamical system is reduced to

$$
\left\{\begin{array}{l}
D^{q} e_{1}=-e_{1} \\
D^{q} e_{2}=-2 \mu e_{2} .
\end{array}\right.
$$

Theorem 3. For any initial conditions, the master and slave defined by the synchronization scheme (17),(18) are globally asymptotically synchronized with the control law (21).

Proof. Applying Laplace transform to system (22), letting $\varepsilon_{1}(s)=L\left(e_{1}(t)\right)$ and $\varepsilon_{2}(s)=L\left(e_{2}(t)\right)$, we obtain

$$
\left\{\begin{array}{l}
s_{1}^{q} \mathcal{E}_{1}(s)-s^{q-1} e_{1}(0)-s^{q-2} e_{1}^{(1)}(0)=-\mathcal{E}_{1}(s) \\
s_{2}^{q} \varepsilon_{2}(s)-s^{q-1} e_{2}(0)-s^{q-2} e_{2}^{(1)}(0)=-2 \mu \varepsilon_{2}(s) .
\end{array}\right.
$$

It follows from Equation (23) that

$$
\left\{\begin{array}{l}
\varepsilon_{1}(s)=\frac{s^{q-1} e_{1}(0)+s^{q-2} e_{1}^{(1)}(0)}{s^{q}+1}, \\
\varepsilon_{2}(s)=\frac{s^{q-1} e_{2}(0)+s^{q-2} e_{2}^{(1)}(0)}{s^{q}+2 \mu} .
\end{array}\right.
$$


From the final-value theorem of the Laplace transform, we have

$$
\left\{\begin{array}{l}
\lim _{t \rightarrow \infty} e_{1}(t)=\lim _{s \rightarrow 0} s \mathcal{E}_{1}(s)=\lim _{s \rightarrow 0} \frac{s^{q-1} e_{1}(0)+s^{q-2} e_{1}^{(1)}(0)}{s^{q}+1}=0, \\
\lim _{t \rightarrow \infty} e_{2}(t)=\lim _{s \rightarrow 0} s \mathcal{E}_{2}(s)=\lim _{s \rightarrow 0} \frac{s^{q-1} e_{2}(0)+s^{q-2} e_{2}^{(1)}(0)}{s^{q}+2 \mu}=0 .
\end{array}\right.
$$

Therefore the synchronization of (17),(18) is achieved under the control law (21).
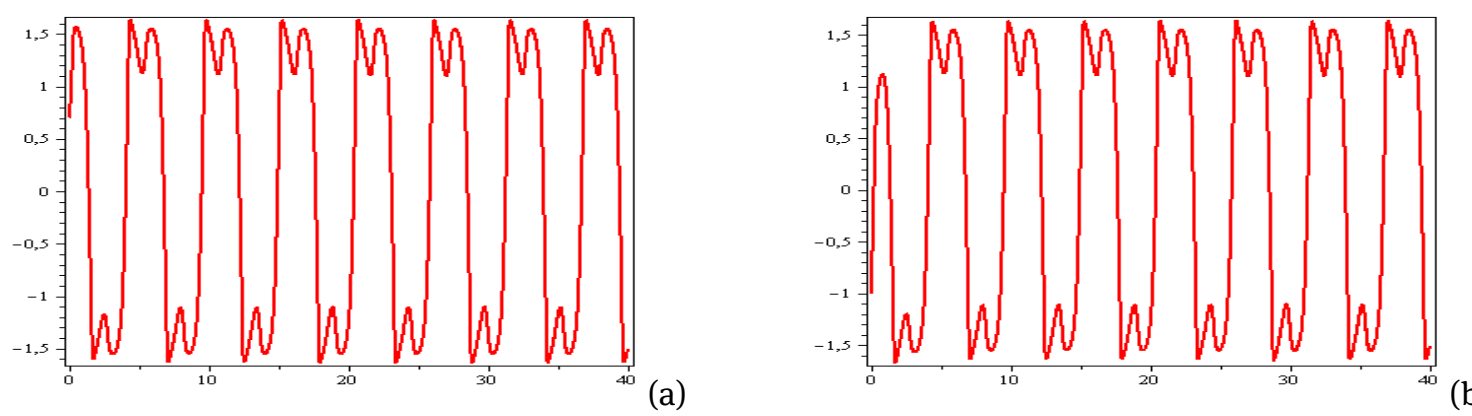

Fig. 3. Time series : (a) $x_{1}(t)$ and (b) $x_{2}(t)$ of (17),(18).
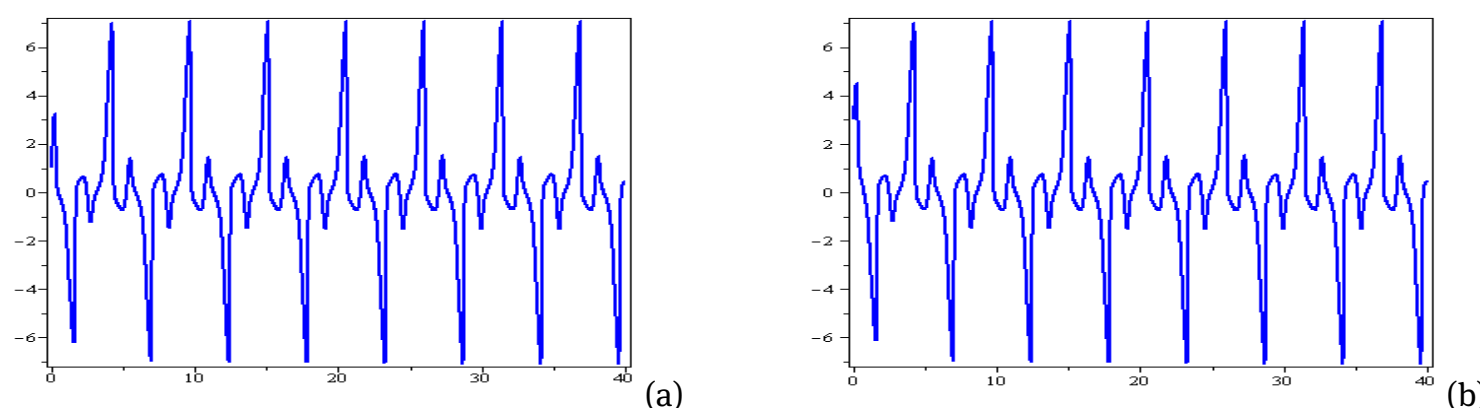

Fig. 4. Time series : (a) $y_{1}(t)$ and (b) $y_{2}(t)$ of (17),(18).
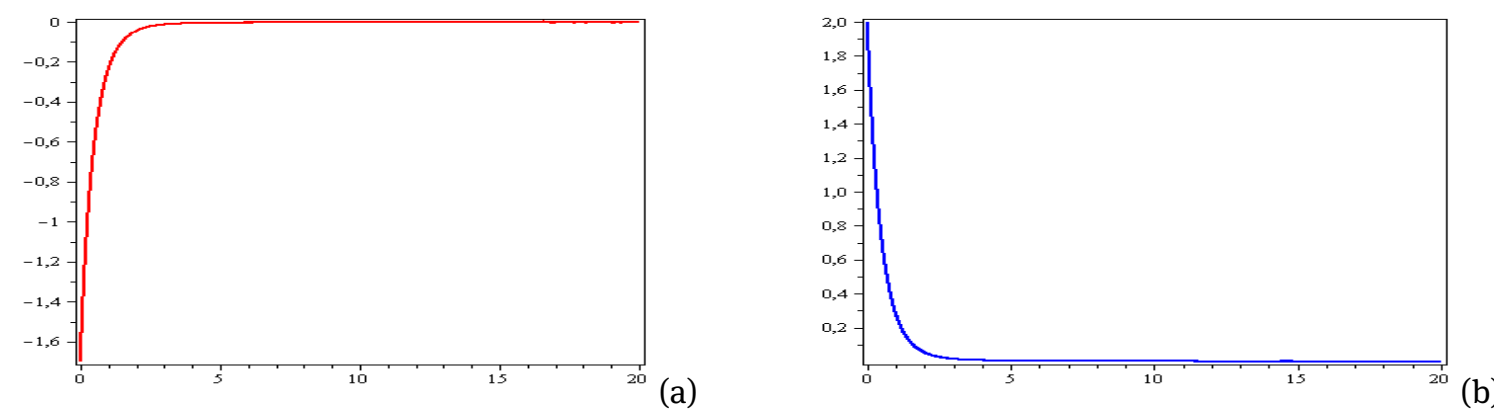

Fig. 5. Evolution of error functions: (a) $e_{1}(t)$, (b) $e_{2}(t)$ of (17),(18). 
Figs.3, 4 and 5 show the simulation result of synchronization of the chaotic systems (17),(18) with initial conditions: $(0.7,1),(0.2,5)$ and $q=0.98$. We remark that the error dynamic system (22) rapidly settles to zero, which means chaos synchronization.

\subsection{Phase-Locked-Loop analogy}

Phase-Locked-Loops (PLL for short) have been applied in various fields. They are increasingly popular in telecommunication systems, wireless systems, power engineering and biological systems. Recently Pham et al. [22] introduced a new model of the FOPLL (Fractional Order Phase-Locked-Loop) and propose control and synchronization methods for it. Motivated by their work, we consider a master-slave synchronization, for system (16) as follows

$$
\left\{\begin{array}{l}
\text { (Master) } \quad D_{t}^{q} X_{1}=\mathbf{A} X_{1}+\mathbf{B} g\left(X_{1}\right)+\mathbf{C}(t), \quad X_{1,0} . \\
\text { (Slave) } \quad D_{t}^{q} X_{2}=\mathbf{A} X_{2}+\mathbf{B} g\left(X_{1}\right)+\mathbf{C}(t)+\mathbf{K e}(t), \quad X_{2,0} .
\end{array}\right.
$$

where $X_{i}=\left(\begin{array}{c}x_{i}(t) \\ y_{i}(t)\end{array}\right)$ for $i=1,2$, and

$$
\left\{\begin{array}{l}
\mathbf{A}=\left(\begin{array}{cc}
0 & 1 \\
-1 & \mu
\end{array}\right), \mathbf{B}=\left(\begin{array}{ll}
0 & 0 \\
0 & 1
\end{array}\right), \mathbf{K}=\left(\begin{array}{ll}
k_{11} & k_{12} \\
k_{21} & k_{22}
\end{array}\right), \\
\mathbf{e}(t)=\left(\begin{array}{c}
e_{1}(t) \\
e_{2}(t)
\end{array}\right), \mathbf{g}\left(X_{1}\right)=\left(\begin{array}{c}
0 \\
-\mu x_{1}^{2} y_{1}+\mu \alpha x_{1}^{4} y_{1}-\beta \mu x_{1}^{6} y_{1}
\end{array}\right) \text { and } \mathbf{C}(t)=\left(\begin{array}{c}
0 \\
E \cos (\omega t)
\end{array}\right) .
\end{array}\right.
$$

where $\mathbf{e}(t)$ is the error synchronization and $\mathbf{K}$ is a matrix to be determined .

The components of the vector error are

$$
\left\{\begin{array}{l}
e_{1}=x_{1}-x_{2} \\
e_{2}=y_{1}-y_{2}
\end{array}\right.
$$

Then the dynamical sychronization error of (26) can be written as

$$
D^{q} \mathbf{e}=(A-\mathbf{k}) \mathbf{e} .
$$

Theorem 4. Systems (26) will approach global synchronization for any initial conditions, with the following control law

$$
\boldsymbol{K e}=\left(\begin{array}{c}
e_{1}+e_{2} \\
e_{1}+4 e_{2}
\end{array}\right),
$$

and $\mu<4$.

Proof. From (28) we have

$$
\left\{\begin{array}{l}
D^{q} e_{1}=-k_{11} e_{1}+\left(1-k_{12}\right) e_{2}, \\
D^{q} e_{2}=-\left(1+k_{21}\right) e_{1}+\left(\mu-k_{22}\right) e_{2} .
\end{array}\right.
$$

The above system has one equilibrium point $(0,0)$, and the Jacobian matrix at this point is

$$
\left(\begin{array}{cc}
-k_{11} & 1-k_{12} \\
-1-k_{21} & \mu-k_{22}
\end{array}\right)
$$


The eigenvalues of the above matrix are:

$$
\lambda_{1,2}=\frac{1}{2}\left(\mu-k_{22}-k_{11} \pm \sqrt{\mu^{2}-2 \mu k_{2}+2 k_{11} \mu+k_{22}^{2}-2 k_{11} k_{22}+k_{11}^{2}-4+4 k_{12}-4 k_{21}+4 k_{21} k_{12}}\right) .
$$

The synchronization of (26) occurs when the matrix $\mathbf{K}$ is chosen appropriately such that

$$
\lim _{t \rightarrow \infty}\|\mathbf{e}(\mathbf{t})\|=\mathbf{0}
$$

hence, we use the condition of stability (7) to find $\mathbf{K}$. Then $\mathbf{K}$ is achieved as

$$
\mathbf{K}=\left(\begin{array}{ll}
1 & 1 \\
1 & 4
\end{array}\right),
$$

and the eigenvalues of (31) become

$$
\lambda_{1}=-1, \quad \lambda_{2}=\mu-4 .
$$

If $\mu<4$, according to Theorem 5 , it is direct to see that the error dynamics converge to the manifold $\left(e_{1}, e_{2}\right)=$ $(0,0)$ as $t \rightarrow \infty$. Consequently the synchronization between two identical systems (26) is achieved via the control law (29).

The effectiveness of the PLL-scheme can be demonstrated through the following numerical simulation presented in Figs.6-8 for $q=0.98$. The initial conditions for the master and slave systems are $(0.7,1)$ and $(-0.2,-1)$ respectively.
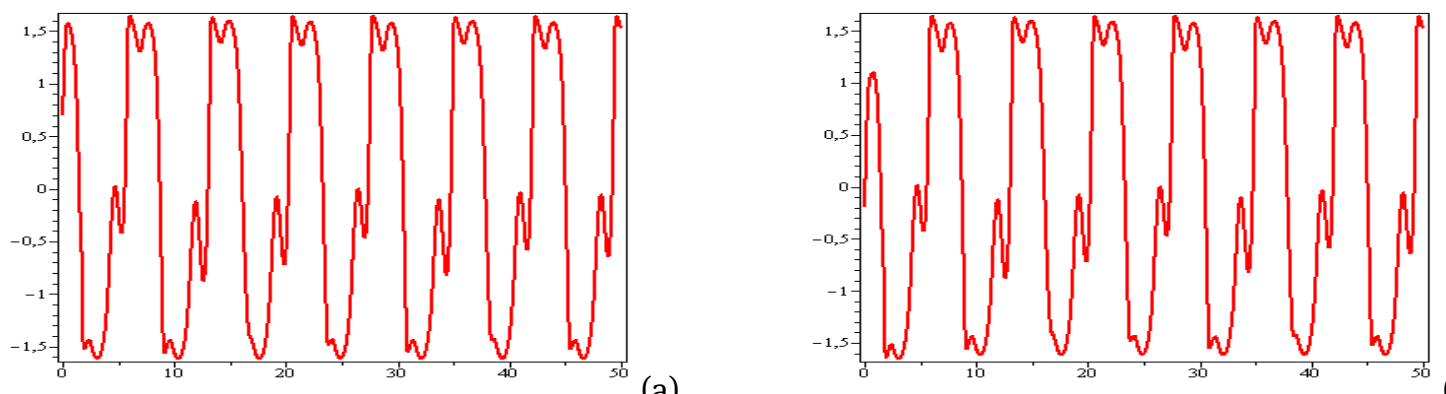

Fig. 6. Time series: (a) $x_{1}(t)$ and (b) $x_{2}(t)$ of (26).

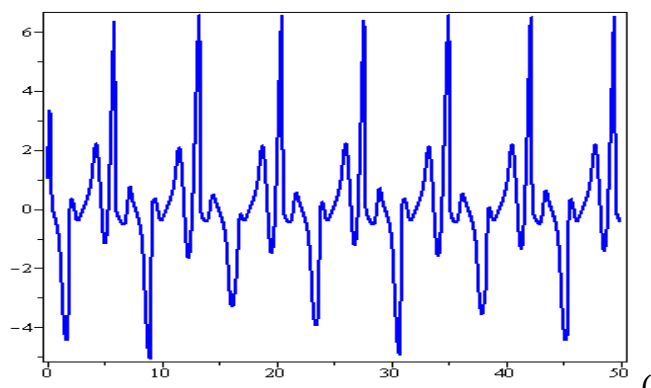

(a)

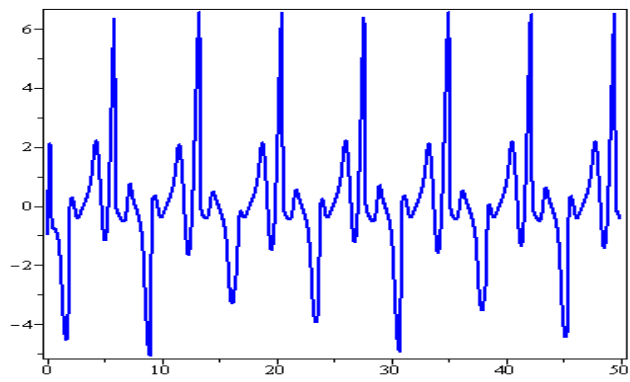

(b)

Fig. 7. Time series: (a) $y_{1}(t)$ and (b) $y_{2}(t)$ of (26). 


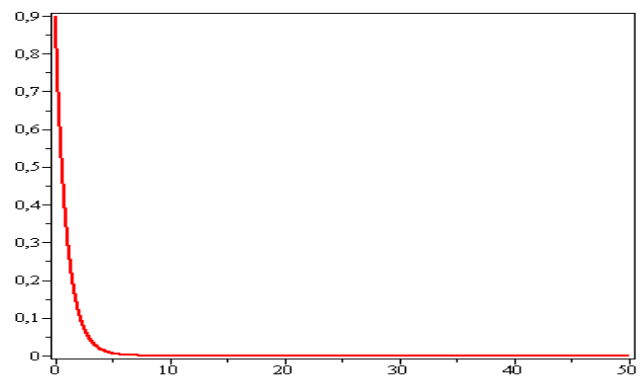

(a)

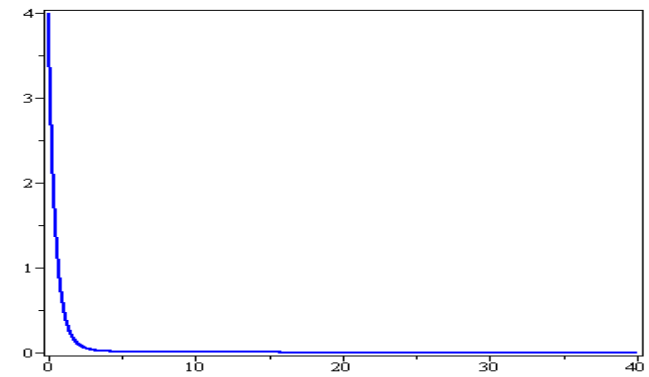

(b)

Fig. 8. Evolution of error functions: (a) $e_{1}(t)$, (b) $e_{2}(t)$ of (26).

\section{Conclusion}

In the present paper, the dynamics for a nonautonomous fractional-order biological system is investigated. Both of the active control and the Phase-Locked-Loop methods are applied to synchronize the fractional order biological system. The corresponding numerical simulations demonstrate the effectiveness of the proposed techniques. We underline that same methods can be used to synchronize a large variety of idential or nonidentical chaotic systems such as: Duffing, Duffing-Holmes, Vander Pol, etc.

\section{References}

[1] E.W. Bai, K. E. Lonngren, Synchronization of two Lorenz systems using active control, Chaos, Solitons Fractals, 8 (1997) pp.51-58.

[2] A. Blokh, C. Cleveland, M. Misiurewicz, Expanding polymodials. Modern dynamical systems and applications, 253-270, Cambridge Univ. Press, Cambridge, 2004.

[3] R.Caponetto, G.Dongola, and L.Fortuna, Fractional order systems: Modeling and control application, World Scientific, Singapore, 2010.

[4] M. Caputo, Linear models of dissipation whose Q is almost frequency independent, J. Roy. Astral.Soc. 13(1967) pp.529539.

[5] A. Chamgoué, R. Yamapi and P. Woafo, Bifurcations in a birhythmic biological system with time-delayed noise, Nonlinear Dynamics. 73, (2013) pp.2157-2173.

[6] K.Diethelm and N.Ford, Analysis of fractional differential equations. Journal of Mathematical Analysis and Applications, 265 (2002) pp.229-248.

[7] K.Diethelm, N.Ford, A.Freed and Y.Luchko, Algorithms for the fractional calculus: a selection of numerical method, Computer Methods in Applied Mechanics and Engineering, 94 (2005) pp.743-773.

[8] H. Frohlich. Long Range Coherence and energy storage in a Biological systems. Int. J. Quantum Chem. 641 (1968) pp.649652.

[9] H. Frohlich, Quantum Mechanical Concepts in Biology. Theoretical Physics and Biology.(1969).

[10] M. Haeri and A. Emadzadeh, Synchronizing different chaotic systems using active sliding mode control, Chaos, Solitons and Fractals. 31 (2007) pp.119-129.

[11] G.He and M.Luo, Dynamic behavior of fractional order Duffing chaotic system and its synchronization via singly active control, Appl. Math. Mech. Engl. Ed., 33 (2012), pp.567-582.

[12] W. Hongwu and M. Junhai, Chaos Controland Synchronization of a Fractional-order Autonomous System, WSEAS Trans. on Mathematics. 11, , (2012) pp. 700-711.

[13] H.G.Kadji, J.B.Orou, R. Yamapi and P. Woafo, Nonlinear Dynamics and Strange Attractors in the Biological System. Chaos Solitons and Fractals. 32 (2007) pp.862-882.

[14] F. Kaiser, Coherent Oscillations in Biological Systems I. Bifurcations Phenomena and Phase transitions in enzymesubstrate reaction with Ferroelectric behaviour. Z Naturforsch A. 294 (1978) pp.304-333.

[15] F. Kaiser, Coherent Oscillations in Biological Systems II. Lecture Notes in Mathematics, 1907. Springer, Berlin, (2007).

[16] E.N. Lorenz, Deterministic nonperiodic flow, J. Atmospheric Science. 20, (1963) pp.130-141. 
[17] L. Lu, C. Zhang and Z.A. Guo, Synchronization between two different chaotic systems with nonlinear feedback control, Chinese Physics, 16(2007) pp.1603-1607.

[18] D. Matignon. Stability results for fractional differential equations with applications to control processing.Proceedings Comp. Eng. Sys. Appl., 963-968, 1996.

[19] K.S Miller and B. Rosso, An Introduction to the Fractional Calculus and Fractional Differential Equations. Wiley, New York 1993.

[20] K. B. Oldham and J. Spanier, The Fractional Calculus. Academic Press, New York, NY, USA, 1974.

[21] O.Olusola, E. Vincent,N. Njah and E. Ali, Control and Synchronization of Chaos in Biological Systems Via Backsteping Design. International Journal of Nonlinear Science . 11 (2011) pp.121-128

[22] V.T.Pham, M.Frasca, R.Caponetto, T.M.Hoang and Luigi Fortuna, Control and synchronization of fractional-order differential equations of phase-locked-loop. Chaotic Modeling and Simulation (CMSIM), 4. (2012) pp.623-631.

[23] L.M. Pecora and T.L. Carroll, Synchronization in chaotic systems, Phys. Rev. Lett. 64, (1990) pp.821-824.

[24] I. Petras, Fractional-Order Nonlinear Systems: Modeling, Analysis and Simulation, Springer, 2011.

[25] A.Pikovsky, Synchronization: A Universal Concept in Nonlinear Sciences, Cambridge University Press 2011.

[26] I.Podlubny, Fractional Differential Equations: Mathematics in Science and Engineering. Academic Press-USA 1999.

[27] S.H. Strogatz, Nonlinear Dynamics and Chaos: With Applications to Physics, Biology, Chemistry and Engineering, Perseus Books Pub., 1994.

[28] A. Ucar, K.E. Lonngren and E.W. Bai, Synchronization of the unified chaotic systems via active control, Chaos, Solitons and Fractals. 27 (2006) pp.1292-97.

[29] Y. Wang, Z.H. Guan and H.O. Wang, Feedback an adaptive control for the synchronization of Chen system via a single variable, Phys. Lett A. 312 (2003) pp.34-40.

[30] G. Zaslavsky, Hamiltonian Chaos and Fractional Dynamics, Oxford University Press, 2008.

Received December 30, 2013; accepted February 14, 2014. 